\title{
The IL9R region contribution in asthma is supported by genetic association in an isolated population
}

\author{
Paula Kauppi ${ }^{1,2}$, Tarja Laitinen ${ }^{2}$, Vesa Ollikainen ${ }^{3}$, Heikki Mannila ${ }^{4}$, Lauri A Laitinen ${ }^{1}$ and \\ Juha Kere $\mathrm{K}^{2,3}$
}

${ }^{1}$ Department of Medicine, Helsinki University Central Hospital; ${ }^{2}$ Department of Medical Genetics; ${ }^{3}$ Finnish Genome Center, University of Helsinki; ${ }^{4} \mathrm{H}$ elsinki University of Technology, Helsinki, Finland

Interleukin 9 (IL9) is involved in mast cell maturation and the enhancement of IgE production by B cells. Furthermore, linkage data in human and mice have suggested that IL9 may contribute to asthma. Since our genetic analysis of the $5 q$ cytokine cluster did not support a genetic role for the IL9 gene, we became interested in the IL9 receptor gene (IL9R) in the pseudoautosomal region. We genotyped markers SDF2 and SDF1 close to the IL9R gene among 289 affected and 368 family-based controls. The results were studied by using linkage, transmission disequilibrium, association and homozygosity analyses. Linkage analyses remained negative, presumably because of our low power for linkage study. However, all the other analyses yielded evidence that the IL9R gene region may have a role in the development of asthma. The sDF2*10 allele was more frequently transmitted than untransmitted to asthmatic offspring (34 vs 16, $\left.P \chi^{2} \leq 0.01\right)$, and it was found homozygotic among asthma patients more often than expected $\left(P_{\text {simul } 2}=0.009\right)$. Also, a specific $X$ chromosomal haplotype, sDF2*10-sDF1*6 associated with asthma (40 vs $\left.\mathbf{7}, \mathbf{P \chi}^{\mathbf{2}}<\mathbf{0 . 0 0 5}, \mathbf{P}_{\text {simul1 }}=\mathbf{0 . 0 4}\right)$. European Journal of Human Genetics $(2000) \mathbf{8}, 788-792$.

Keywords: asthma; association; interleukin 9 receptor; pseudoautosomal region

\section{Introduction}

The interleukin 9 receptor (IL9R) was the first gene to be identified in the pseudoautosomal region $(320 \mathrm{~kb})$ of the $\mathrm{Xq}$ and $\mathrm{Yq}$ chromosomes. The genes in the pseudoautosomal region escape $X$ inactivation and are expressed by both $X$ and $Y$ chromosomes. ${ }^{1}$ Four pseudogenes of the IL9R have been located on chromosomes $9 q, 10 p, 16 p$ and $18 p$ without any functional activity. ${ }^{2}$ The IL9R ( $17 \mathrm{~kb}, 11$ exons) encodes a ligand specific alpha chain ( 522 amino acids and $64 \mathrm{kDa}$ ) for the heterodimeric receptor ( $\alpha$ and $\gamma$ subunits). The receptor is expressed by a variety of haematopoietic cells such as T cells, mast cells, and macrophages. Other interleukin receptors such as IL2, IL4, IL7, and IL15 share the $\gamma$ chain of the receptor $^{3}$ which might explain partially overlapping functions of these interleukins. ${ }^{4}$

The gene for IL9 is located on chromosome 5q31-q33 together with an interleukin gene cluster (IL3, IL4, IL5, IL 13,

Correspondence: Dr Paula Kauppi MD, Department of Medicine, Division of Pulmonary Medicine, Helsinki University Central Hospital, PO Box 340, Fin-00029 Helsinki, Finland. Fax: +358947174049; Tel: +358947173557; E-mail: paula.kauppi@pp.fimnet.fi

Received 28 February 2000; revised 5 June 2000; accepted 20 June 2000
GM-CSF). Quantitative traits (IgE and bronchial hyperreactivity) associated with asthma have been reported to show linkage to this region., ${ }^{5,6}$ However, linkage to the $5 q$ region has not been confirmed in all populations studied, ${ }^{7,8}$ suggesting population heterogeneity in the molecular genetic background of the traits.

IL9 has many biological effects and some of them synergise with IL4 in IgE production. In addition, IL9 induces growth and maturation of mast cells. The IL9 gene has also been linked to bronchial hyper-reactivity in mice, and a reduced expression of IL9 was shown in lung tissue of a hyporesponsive mouse strain, ${ }^{9}$ while an IL 9 transgenic mouse has elevated serum total IgE level, bronchial hyper-responsiveness and eosinophilia of the airways in response to an antigen challenge. ${ }^{10}$ In human, linkage studies have not implicated any gene in $5 q$, although Doull et al have reported some evidence of an association of the IL9 gene to high serum total IgE level. ${ }^{11}$ Among the Finns, a single nucleotide polymorphism in the IL 9 gene was found (T113M), but there was no association with high serum total IgE level or asthma. $^{7}$ 
Increased evidence of the role of IL9 in atopic disorders and having found no association between asthma or high IgE and the interleukin gene cluster in $5 q$ among the Finns, raised the question of other molecules in the IL9 pathway. Polymorphism in the IL4RA gene has been reported to associate with atopy. ${ }^{12}$ In the present study, we have analysed two polymorphic markers close to the IL9R gene with regard to asthma and IgE level with different analytical approaches.

\section{Material and methods \\ Subjects}

The study sample consists of 202 nuclear families with 657 study individuals recruited in the Kainuu province of Finland. The inclusion criteria for probands were: selfreported asthma diagnosed by a physician, nuclear family willing to participate, and parents or grandparents born in Kainuu. Our procedure for verification of the diagnosis have been reported in detail. ${ }^{13}$ Both the probands and their family members gave written informed consent. Blood samples were collected both for the DNA analysis and for measuring total serum IgE (Diagnostics CAP FEIA, Kabi Pharmacia, Sweden). The ethical committees of the Department of Medical Genetics, University of Helsinki and the Kainuu Central Hospital have approved the study.

\section{Markers and genotyping}

DNA was extracted from blood leukocytes by a standard nonenzymatic method. The polymorphic markers sDF1 (DXYS154) (heterozygosity 0.73) and SDF2 (DXS1108) (heterozygosity 0.70 ) were typed by using published primer sequences. ${ }^{14}$ Physical locations of the markers at the boundary of $X$ specific and $X Y$ homology regions, not more than $100 \mathrm{~kb}$ apart, have previously been established. ${ }^{15}$ The IL9R gene is located distal to the markers, close to the telomere $(20-30 \mathrm{~kb}){ }^{2}$ The PCR assays contained $50 \mathrm{ng}$ of genomic DNA, $0.4 \mathrm{~mm}$ of primer mix, $0.3 \mathrm{U}$ of DNA polymerase (Dynazyme, Finnzymes, Finland) and $0.2 \mathrm{~mm}$ of each dNTP in a total volume of $20 \mu \mathrm{l}$. The PCR conditions were $3 \mathrm{~min}$ at $93^{\circ} \mathrm{C}$, followed by 30 cycles each of $15-30 \mathrm{~s}$ at $93^{\circ} \mathrm{C}, 15-30 \mathrm{~s}$ at $50-59^{\circ} \mathrm{C}, 15-30 \mathrm{~s}$ at $72^{\circ} \mathrm{C}$ and final $8 \mathrm{~min}$ at $72^{\circ} \mathrm{C}$. The samples were electrophoresed on polyacrylamide gels and alleles were visualised by silver staining.

\section{Statistical analyses and power estimations}

Single-point non-parametric linkage analysis and transmission disequilibrium test (TDT) were done for both phenotypes, asthma and high serum IgE level, using the computer package GENEHUNTER. ${ }^{16}$

In allele and haplotype association studies, a chromosome was marked as 'trait-associated' if it occurred in any affected family member and as 'control' if it occurred only in unaffected family members. ${ }^{17}$ Every chromosome in each family was counted only once. The haplotypes were constructed by hand. The allele and haplotype associations were initially analysed with chi-square test $\left(P \chi^{2}\right)$. Since the $P \chi^{2}$ test was performed multiple times, a permutation test $\left(\mathrm{P}_{\text {simul } 1}\right)$ was used to estimate the overall significance: ${ }^{7}$ the haplotypes were fixed, while the phenotypes of the chromosomes were randomised. The proportion of 1000 such iterations where a stronger association was found than in actual data showed the simulated $P_{\text {simul }}$ value of the observation. Comparison was done by using $\mathrm{P}_{\chi}{ }^{2}$ values of the most extreme association in simulated and actual data.

The simulations for homozygosity testing $\left(\mathrm{P}_{\text {simul } 2}\right)$ of the sDF2 alleles were carried out with females only. All unrelated affected females were considered and their observed alleles were used for simulations. Then, in each of 100000 iterations, random pairs of these alleles were formed, and the number of simulated homozygous chromosome pairs was counted. The iterations provided a distribution for the number of expected homozygotes under the null hypothesis of no excess homozygosity, treating the number of alleles fixed. Finally, the observed number of homozygotes was compared with this distribution to obtain the $\mathrm{P}_{\text {simul2 }}$ value.

We performed additional simulations (Simul3) also to assess the power of the sample to detect the presence of an ancestral chromosome carrying susceptibility to a trait. ${ }^{7} \mathrm{~A}$ two-locus haplotype was chosen be present at frequency $\mathrm{F}$ (5\%, 10\%, 15\% and 20\%) among the affected chromosomes of the data set; 10000 iterations were done for each $\mathrm{F}$ and the power to detect association was measured by the fraction of the replicates in which the haplotype showed $P \chi^{2}=0.05$.

\section{Results \\ Non-parametric single-point linkage study}

Linkage was studied in 80 sib pairs and 62 cousin pairs for asthma and for high serum total IgE level (84 families). Both phenotypes were analysed as qualitative traits (asthmatic vs non-asthmatic and serum $\mathrm{IgE}>100 \mathrm{kU} / \mathrm{L}$ vs serum $\operatorname{lgE} \leq 100 \mathrm{kU} / \mathrm{L})^{6,18}$ Non-parametric linkage score (NPL) remained negative for both markers and for both phenotypes with an information content of $34-46 \%$, but did not allow exclusion.

\section{Transmission disequilibrium test}

TDT was analysed first in all the families where the child was affected and both parents had heterozygous genotypes (Table1). In men the genotype for sDF2 was marked as $Y$ (for $Y$ chromosome) and the hemizygous allele (for $X$ chromosome). The allele SDF2*10 was transmitted to 34 and untransmitted to 16 asthmatic offspring ( $P \chi^{2} \leq 0.01$ ) (Table1). When 2-marker haplotypes were analysed the excess of SDF2*10 originated mainly from two parental $X$ chromosomes: sDF2*10-sDF1*6 (11transmitted vs 3 chromosomes untransmitted, $\mathrm{P} \chi^{2}=0.03$ ) and SDF2*10SDF1*1 (5transmitted vs 0 chromosomes untransmitted, $\left.P \chi^{2}=0.03\right)$. 
Table 1 Comparison of transmitted/untransmitted parental chromosomes to affected offspring and transmitted/ untransmitted matemal chromosomes to affected daughters

\begin{tabular}{|c|c|c|c|c|}
\hline \multirow[b]{2}{*}{ Marker } & \multicolumn{2}{|c|}{ Parental } & \multicolumn{2}{|c|}{ Maternal } \\
\hline & Trans & Untrans & Trans & Untrans \\
\hline sDF2 allele *3 & 13 & 10 & 6 & 6 \\
\hline sDF2 allele $* 4$ & 60 & 50 & 12 & 23 \\
\hline sDF2 allele *5 & 37 & 43 & 13 & 15 \\
\hline sDF2 allele *6 & 1 & 0 & & \\
\hline sDF2 allele *10 & 34 & $16^{b}$ & 17 & $4^{a}$ \\
\hline Y chromosome & 30 & $58^{b}$ & & \\
\hline sDF1 allele $* 1$ & 5 & $0^{c}$ & 2 & 0 \\
\hline sDF1 allele *2 & 5 & 8 & 1 & 2 \\
\hline sDF1 allele $* 3$ & 9 & 5 & 3 & 3 \\
\hline sDF1 allele $* 4$ & 30 & 40 & 6 & 8 \\
\hline sDF1 allele *5 & 22 & 24 & 12 & 7 \\
\hline sDF1 allele $* 6$ & 50 & 37 & 11 & 13 \\
\hline sDF1 allele *7 & 4 & 7 & 1 & 2 \\
\hline sDF1 allele *9 & 4 & 6 & 1 & 2 \\
\hline sDF1 allele *10 & 0 & 2 & & \\
\hline
\end{tabular}

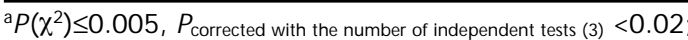

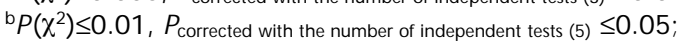

${ }^{\mathrm{C}} \mathrm{P}\left(\chi^{2}\right) \leq 0.05, \mathrm{P}_{\text {corrected with the number of independent tests (8); }}$ ns (not significant).

TDT was then analysed separately for paternal and maternal transmission to sons and daughters. As expected, the allele spectrum of the pseudoautosomal marker (sDF1) in $Y$ chromosomes was narrower than in $\mathrm{X}$ chromosomes, reflecting a founder effect. ${ }^{19}$ The analysis also showed female overrepresentation among the patients (67\%). These potential confounding factors are avoided when solely maternal transmission to affected daughters is considered (Table1). In this test, the haplotype sDF2*10-sDF1*6 was transmitted ${ }^{5}$ more frequently to affected daughters than untransmitted $(0),\left(P \chi^{2}=0.03\right)$. For high serum IgE, the TDT results were not significant.

\section{Association study}

The allele SDF2*10 in $X$ chromosomes was found more frequently in asthma than in control chromosomes ( $28 \%$ vs $\left.20 \%, P \chi^{2}=0.05\right)$ (Table2). To correct the result for multiple testing, 1000 permutations were done ( $P_{\text {simul } 1}$, see methods). In 176 such permutations for the asthma phenotype, the most extreme allele association found in simulated data was stronger than in actual data $\left(P_{\text {simul } 1}=0.18\right)$. The allele frequencies of the marker SDF1 were equally distributed in affected and control chromosomes.

Haplotype association analysis was performed on X chromosomes. For asthma, the haplotype sDF2*10-sDF1*6 was found more frequently in affected chromosomes than in control chromosomes ( 40 affected vs 7 control chromosomes $\left(\mathrm{P} \chi^{2}<0.005\right)$ (Table3). In the permutation test, only 40 out of 1000 iterations provided a haplotype association more extreme than in actual data $\left(P_{\text {simul } 1}=0.04\right)$. Analysed separately for male and female $X$ chromosomes, the association was significant for females (34 affected haplotypes and 6 controls) by a $\mathrm{P} \chi^{2}$ test $(\mathrm{P}<0.05)$. For high IgE level, no allele or haplotype associations were found (Table2). By power simulations (Simul3), our power to show haplotype association for high total serum IgE level was 99-93\%, if the frequency of a susceptibility haplotype among affected chromosomes $(F)$ is between 0.15 and 0.20 . When $F=0.10$ or $\mathrm{F}=0.05$ the corresponding power to detect the association

Table 2 Allele associations found in asthma and high serum IgE with the markers sDF2 and sDF1. For HWE, the expected number of all heterozygous individuals is $70.9 \%$ for SDF2 and $71.8 \%$ for sDF1. The observed numbers did not differ significantly from the expected

\begin{tabular}{|c|c|c|c|c|c|c|c|c|}
\hline \multirow[b]{2}{*}{ Marker } & \multicolumn{4}{|c|}{ Asthma } & \multicolumn{4}{|c|}{ High IgE } \\
\hline & Affected \% & $\mathrm{n}$ & Control \% & $\mathrm{n}$ & Affected \% & $\mathrm{n}$ & Control \% & $n$ \\
\hline sDF2 allele $* 2$ & 0.3 & 1 & 0 & 0 & 0 & 0 & 0.3 & 1 \\
\hline sDF2 allele $* 3$ & 5.5 & $1 \overline{9}$ & 6.6 & 12 & 6.6 & 16 & 6.8 & $2 \overline{2}$ \\
\hline sDF2 allele $* 4$ & 38.8 & 133 & 42.9 & 78 & 39.3 & 96 & 39.9 & 129 \\
\hline sDF2 allele *5 & 26.5 & 91 & 29.7 & 54 & 29.9 & 73 & 26.3 & 85 \\
\hline sDF2 allele *6 & 0.6 & 2 & 1.1 & 2 & 0.8 & 2 & 0.6 & 2 \\
\hline sDF2 allele *9 & 0.6 & 2 & 0 & 0 & 0 & 0 & 0.6 & 2 \\
\hline sDF2 allele *10 & $27.7^{\mathrm{c}}$ & 95 & 19.8 & 36 & 23.4 & 57 & 25.4 & 82 \\
\hline sDF2 total ${ }^{\mathrm{a}}$ & 100 & 343 & 100 & 182 & 100 & 244 & 100 & 323 \\
\hline sDF1 allele $* 1$ & 1.4 & 6 & 0.4 & 1 & 1.6 & 5 & 1.4 & 6 \\
\hline sDF1 allele $* 2$ & 3.6 & 15 & 5.7 & 15 & 3.5 & 11 & 4.8 & 20 \\
\hline sDF1 allele $* 3$ & 4.8 & 20 & 3.4 & 9 & 4.7 & 15 & 4.3 & 18 \\
\hline sDF1 allele $* 4$ & 27.0 & 113 & 29.8 & 78 & 26.6 & 84 & 28.9 & 121 \\
\hline sDF1 allele *5 & 15.5 & 65 & 16.4 & 43 & 15.2 & 48 & 16.3 & 68 \\
\hline sDF1 allele *6 & 42.0 & 176 & 38.2 & 100 & 41.1 & 130 & 38.3 & 160 \\
\hline sDF1 allele $* 7$ & 3.3 & 14 & 3.8 & 10 & 4.7 & 15 & 3.8 & 16 \\
\hline sDF1 allele *10 & 0.2 & 1 & 0.4 & 1 & 0.3 & 1 & 0.2 & 1 \\
\hline sDF1 total ${ }^{\mathrm{b}}$ & 100 & 419 & 100 & 262 & 100 & 316 & 100 & 418 \\
\hline
\end{tabular}

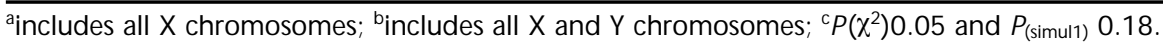


Table 3 All X-chromosomal haplotypes for the phenotype of asthma with 343 affected and 182 control chromosomes. Haplotypes with a number less than 10 have been pooled together $\left(\mathrm{sDF}^{*} \mathrm{z}\right)$

\begin{tabular}{|c|c|c|c|c|c|}
\hline \multirow[b]{2}{*}{ Haplotype } & \multicolumn{2}{|c|}{ Affected } & \multicolumn{2}{|c|}{ Control } & \multirow[b]{2}{*}{$P \chi^{2}$} \\
\hline & $\mathrm{N}$ & $\%$ & $\mathrm{~N}$ & $\%$ & \\
\hline sDF2*2-sDF1*z & 1 & 0.3 & 0 & 0 & ns \\
\hline sDF2*3-sDF1*6 & 10 & 2.9 & 4 & 2.2 & ns \\
\hline sDF2*3-sDF1*z & 9 & 2.6 & 8 & 4.4 & ns \\
\hline sDF2*4-sDF1*3 & 14 & 4.1 & 4 & 2.2 & ns \\
\hline sDF2*4-sDF1*4 & 18 & 5.2 & 16 & 8.8 & ns \\
\hline sDF2*4-sDF1*5 & 14 & 4.1 & 8 & 4.4 & ns \\
\hline sDF2*4-sDF1*6 & 71 & 20.7 & 42 & 23.1 & ns \\
\hline sDF2*4-sDF1*7 & 10 & 2.9 & 3 & 1.6 & ns \\
\hline sDF2*4-sDF1*z & 6 & 1.7 & 5 & 2.7 & ns \\
\hline sDF2*5-sDF1*4 & 21 & 6.1 & 11 & 6.0 & ns \\
\hline sDF2*5-sDF1*5 & 20 & 5.8 & 8 & 4.4 & ns \\
\hline sDF2*5-sDF1*6 & 35 & 10.2 & 27 & 14.8 & ns \\
\hline sDF2*5-sDF1*z & 15 & 4.4 & 8 & 4.4 & ns \\
\hline sDF2*6-sDF1*z & 2 & 0.6 & 2 & 1.1 & ns \\
\hline sDF2*9-sDF1*z & 2 & 0.6 & 0 & 0 & ns \\
\hline sDF2*10-sDF1*4 & 25 & 7.3 & 11 & 6.0 & ns \\
\hline sDF2*10-sDF1*5 & 18 & 5.2 & 13 & 7.1 & ns \\
\hline sDF2*10-sDF1*6 & $40^{a}$ & 11.7 & 7 & 3.8 & $\lessdot 0.005$ \\
\hline sDF2*10-sDF1*z & 12 & 3.5 & 5 & 2.7 & ns \\
\hline
\end{tabular}

$\operatorname{ap}\left(\chi^{2}\right) \prec 0.005$ and $\mathrm{P}_{\text {(simul1) }} 0.04$.

decreases ( $67 \%$ and $18 \%$, respectively). Power to detect a hapl otype association for the asth ma phenotype was similar: $97 \%(F=0.20), 81 \%(F=0.15), 49 \%(F=0.10)$, and $6 \%$ $(\mathrm{F}=0.05)$

\section{Homozygosity testing}

If a marker allele is associated with a disease gene, homozygosity for the allele should associate with the disease even more strongly. When testing this hypothesis with a randomization test $\left(\mathrm{P}_{\text {simul } 2}\right)$, an excess of homozygosity was found for the SDF2*10 allele and asthma phenotype (Table4). Among the asthma patients, 13 sDF2*10 homozygous individuals were observed, whereas the average expected from the simulation was $7.7\left(P_{\text {simul } 2}=0.009\right)$. For the controls, the number of homozygous individuals was only 3. The overall observed homozygosity for both markers did not differ from

Table 4 Observed heterozygosity and homozygosity for the marker sDF2 compared to expected homozygosity based on 100000 simulations among the asthma patients

\begin{tabular}{|c|c|c|c|c|c|}
\hline Marker & Freq. & $\begin{array}{l}\quad \text { Asthr } \\
\text { Observed } \\
\text { hetero- } \\
\text { zygosity }\end{array}$ & $\begin{array}{l}\text { na patients } \\
\text { Observed } \\
\text { homo- } \\
\text { zygosity }\end{array}$ & $\begin{array}{l}\text { Expected } \\
\text { homo- } \\
\text { zygosity }\end{array}$ & $\mathrm{P}_{(\text {simul2) }}$ \\
\hline sDF2*3 & 0.062 & 10 & 1 & 0.3 & 0.2917 \\
\hline sDF2*4 & 0.344 & 34 & 16 & 11.2 & 0.0252 \\
\hline sDF2*5 & 0.286 & 37 & 9 & 7.7 & 0.3426 \\
\hline sDF2*6 & 0.010 & 2 & 0 & 0.0 & 1.0000 \\
\hline sDF2*9 & 0.010 & 0 & 1 & 0.0 & 0.0044 \\
\hline sDF2*10 & 0.286 & 29 & 13 & 7.7 & 0.0090 \\
\hline
\end{tabular}

the expected, confirming that the study population is in Hardy-Weinberg equilibrium (HWE) (Table2).

\section{Discussion}

Recent studies in human and in mice have suggested IL9-mediated effects to be relevant for the development of atopic disorders. To study the counterpart of the ligand, the IL9R gene, we used two polymorphic markers not more than $400 \mathrm{~kb}$ centromeric of the IL9R gene instead of a polymorphic repeat in the coding region of the IL $9 R$ gene, since IL $9 R$ pseudogenes may affect the interpretation of the intragenic polymorphism. ${ }^{1}$ Among the Kainuu population, linkage disequilibrium has previously been shown to extend regions much longer than the region studied here. ${ }^{20}$ So, we have no reason to believe that the markers would not be in association with possible susceptibility alleles of the IL9R gene.

Holroyd et al have previously found linkage between asthma and this same PAR region with the best NPL score for the marker DXYS154 (SDF1). ${ }^{21}$ In our study, association, transmission disequilibrium and homozygosity testing yielded evidence that the IL9R region may have biological significance for the development of asthma. These methods can be more powerful than linkage when multifactorial diseases are studied. ${ }^{22}$ Having a young founder population, our sample was designed to have high power for haplotype association if a susceptibility allele is present in at least $15 \%$ of the affected chromosomes, whereas our power for genetic linkage study was much lower. Both TDT and haplotype association recognised the same haplotype (sDF2*10-sDF1*6) which was 2.1 times more frequent among haplotype positive affected females than haplotype positive unaffected females. Thus, we propose that sDF2*10-sDF1*6 is a marker of an ancestral chromosome carrying a functional variant of the IL9R gene, although the effect of the gene on the asthma phenotype seems relatively minor at population level. The most important confounding factor which could mislead our conclusions is population admixture. However, we could exclude close relationships between the families and verify the origin of the families by genealogical studies. ${ }^{7}$ In addition, no deviation from HWE was detected in this chromosomal region (Table2) or other regions studied previously. $^{7}$

The biological significance of a weak association is difficult to interpret especially in complex disorders. In many cases, the reported association has not been found in any other population and the differences between the results have been explained by genetic heterogen eity between the populations. Supporting a role for IL9R, a splice variant in the coding region of the IL9R gene has been reported to alter the binding of IL $9^{23}$ which demonstrates the importance of a functional receptor in IL9 signaling. This finding together with the reported positive linkage between asthma and the $\mathrm{Xq} / \mathrm{Yq}$ pseudoautosomal region ${ }^{21}$ is supported by our results of the same region. All this speaks for the relative importance of the 
IL9R gene region in genetic control for the susceptibility to asthma.

\section{Acknowledgements}

The authors wish to thank Dr Mark Daly for allowing use of the new version of Genehunter computer package. We thank Ms Liisa Rajasalo, Ms Päivikki Pajunen and their staff at Kainuu Central Hospital for collection of the samples, and Ms Siv Knaappila and Dr Tarja Ruotsalainen for their genotyping work. This study was supported by the Academy of Finland, Helsinki University Hospital Research Funds, Ida Montin Foundation, Sigrid Juselius Foundation, Finnish Anti-Tuberculosis Association Foundation, and by CEC/BIOMED2 (BMH4-CT97-2486).

\section{References}

1 Vermeesch JR, Petit P, Kermouni A, Renauld JC, Van Den Berghe $H$, Marynen $P$ : The IL-9 receptor gene, located in the $X q / Y q$ pseudoautosomal region, has autosomal origin, escapes $X$ inactivation and is expressed from the Y. Hum Mol Genet 1997; 6: 1-8.

2 Kermouni A, van Roost E, Arden KC et al: The IL-9 receptor (IL9R) gene: genomic structure, chromosomal localization in the pseudoautosomal region of the long arm of the sex chromosomes, and identification of the IL9R pseudogenes at 9qter, 10pter, 16pter, and 18pter. Genomics 1995; 29: 371-382.

3 Zhu YX, Sun HB, Lik-Shing Tsang $M$ et al: Critical cytoplasmic domains of human interleukin-9 receptor alpha chain in interleukin-9-mediated cell proliferation and signal transduction. J Biol Chem 1997; 272: 21334-21340.

4 Demoulin JP, Renauld JC: Interleukin 9 and its receptor: an overview of structure and function. Intern Rev Immunol 1998; 16: 345-364.

5 Marsh DG, Neeley JD, Breazeale DR et al: Linkage analysis of IL4 and other chromosome $5 q 31.1$ markers and total serum immunoglobulin E concentrations. Science 1994; 264: 1152-1156.

6 Postma DS, Bleecker ER, Amelung PJ et al: Genetic susceptibility to asthma - bronchial hyperresponsiveness co-inherited with a major gene for atopy. N Engl J Med 1995; 333: 894-900.

7 Laitinen T, Kauppi P, Ignatius J et al: Genetic control of serum IgE levels and asthma: linkage and linkage disequilibrium studies in and isolated population. Hum Mol Genet 1997; 6: 2069-2076.

8 Blumenthal MN, Wang Z, Weber JL, Rich SS: Absence of linkage between $5 q$ markers and serum IgE levels in four large atopic families. Clin Exp Allergy 1996; 26: 892-896.

9 Nicolaides NC, Holroyd KJ, Ewart SL et al: Interleukin 9: A candidate gene for asthma. Proc Natl Acad Sci USA 1997; 94: 13175-13180.
10 McLane MP, Haczku A, van de Rijn M et al: Interleukin-9 promotes allergen-induced eosinophilic inflammation and airway hyperresponsiveness in transgenic mice. Am J Respir Cell Mol Biol 1998; 19: $713-720$.

11 Doull IJM, Lawrence S, Watson M et al: Allelic association of gene markers on chromosomes $5 \mathrm{q}$ and $11 \mathrm{q}$ with atopy and bronchial hyperresponsiveness. Am J Respir Crit Care Med 1996; 153: 1280-1284.

12 Hershey GKK, Friedrich MF, Esswein LA, Thomas ML, Chatila TA: The association of atopy with a gain-of-function mutation in the alpha subunit of the interleukin-4 receptor. N Engl J Med 1997; 337: 1720-1725.

13 Kauppi P, Laitinen LA, Laitinen $\mathrm{H}$, Kere J, Laitinen $\mathrm{T}$ : Verification of self-reported asthma and allergy in subjects and their family members volunteering for gene mapping studies. Resp Med 1998; 92: 1281-1288.

14 Freije D, Helms C, Watson MS, Donis-Keller $\mathrm{H}$ : Identification of a second pseudoautosomal region near the $\mathrm{Xq}$ and $\mathrm{Yq}$ telomeres. Science 1992; 258: 1784-1787.

15 Kvaløy K, Galvagni F, Brown WRA: The sequence organization of the long arm pseudoautosomal region of the human sex chromosomes. Hum Mol Genet 1994; 3: 771-778.

16 Kruglyak L, Daly MJ, Reeve-Daly MP, Lander ES: Parametric and nonparametric linkage analysis: a unified multipoint approach. Am J Hum Genet 1996; 58: 1347-1363.

17 Thomson G: Mapping disease genes: Family-based association studies. Am J Hum Genet 1995; 57: 487-498.

18 Burrows B, Martinez FD, Halonen M, Barbee RA, Cline MG: Association of asthma with serum IgE levels and skin-test reactivity to allergens. N Engl J Med 1989; 320: 271-277.

19 Sajantila A, Salem AH, Savolainen P, Bauer K, Gierig C, Paabo S: Paternal and maternal DNA lineages reveal a bottleneck in the founding of the Finnish population. Proc Natl Acad Sci USA 1996; 93: 12035-12039.

20 Höglund $\mathrm{P}$, Sistonen $\mathrm{P}$, Norio $\mathrm{R}$ et al: Fine mapping of the congenital chloride diarrhea gene by linkage disequilibrium. Am J Hum Genet 1995; 57: 95-102.

21 Holroyd KJ, Martinati LC, Trabetti E et al: Asthma and bronchial hyper-responsiveness linked to the $X Y$ long arm pseudoautosomal region. Genomics 1998; 52: 233-235.

22 Yu CE, Payami H, Olson JM et al: The apolipoprotein E/Cl/CII gene cluster and late-onset Alzheimer disease. Am J Hum Genet 1994; 54: 631-642.

23 Grasso L, Huang $M$, Sullivan $C D$ et al: Molecular analysis of human interleukin-9 receptor transcripts in peripheral blood mononuclear cells. J Biol Chem 1998; 273: 24016-24024. 